\title{
NONSELFADJOINT FOURTH ORDER DIFFERENTIAL EQUATIONS WITH CONJUGATE POINTS
}

\author{
BY KURT KREITH
}

Communicated by Murray Protter, April 2, 1974

Much of the classical Sturm oscillation theory extends to selfadjoint differential equations of order $2 n$ if the notion of "oscillation" is formulated in terms of conjugate points determined by nontrivial solutions with $n$th order zeros at two distinct points. The standard techniques for achieving such generalizations also allow one to establish disconjugacy criteria in the nonselfadjoint case (see [1] - [4]), but I know of no corresponding criteria for the existence of conjugate points when the equation under consideration is not selfadjoint.

The purpose of this note is to sketch a technique which deals with the sufficiently regular general real fourth order equation

$$
l[y] \equiv\left(p_{2}(t) y^{\prime \prime}-q_{2}(t) y^{\prime}\right)^{\prime \prime}-\left(p_{1}(t) y^{\prime}-q_{1}(t) y\right)^{\prime}+p_{0}(t) y=0,
$$

and establishes the existence of a $\beta>\alpha$ such that

$$
y(\alpha)=y^{\prime}(\alpha)=0=y(\beta)=y^{\prime}(\beta)
$$

is satisfied by a nontrivial solution $y(t)$ of (1). A detailed proof will appear elsewhere.

We begin with an oscillation preserving transformation used by the author [5] to eliminate the third order term $\left(q_{2}(t) y^{\prime}\right)^{\prime \prime}$. Generalizing upon a technique used by Whyburn [6] for selfadjoint equations, one can then obtain a representation of (1) in the form

$$
y^{\prime \prime}=a(t) y+b(t) x, \quad x^{\prime \prime}=c(t) y+d(t) x
$$

where $b(t)=1 / p_{2}(t)>0$, and the formulas for the other coefficients of (2) are given in [5]. The system (2) allows an obvious dynamical interpretation in terms of a particle of unit mass moving in the $x, y$-plane. By an

AMS (MOS) subject classifications (1970). Primary 34C10.

Key words and phrases. Disconjugacy, oscillatory.

Copyright $\odot$ 1974, American Mathematical Society 
appropriate normalization, solutions of (1) satisfying $y(\alpha)=y^{\prime}(\alpha)=0$ may be represented by a one-parameter family of trajectories $C\left(v_{0}\right)$ determined by the initial conditions

$$
y(\alpha)=y^{\prime}(\alpha)=0 ; \quad x(\alpha)=1 ; \quad x^{\prime}(\alpha)=v_{0}
$$

We therefore seek an appropriate value of $v_{0}$ such that a particle fired from $(1,0)$ with an initial velocity $v_{0}$ in the direction of the positive $x$-axis will, at some later time $t=\beta$, again move tangent to the $x$-axis. Labelling the open quadrants of the $x, y$-plane I, II, III, and IV, a topological argument shows that the following four conditions on the force field determined by (2) suffice to assure existence of such a trajectory:

If for some $t_{0} \geqslant \alpha$ the quantities $y\left(t_{0}\right), y^{\prime}\left(t_{0}\right), x\left(t_{0}\right)$

(A) and $x^{\prime}\left(t_{0}\right)$ are all nonnegative (but not all zero), then $y(t), y^{\prime}(t), x(t)$ and $x^{\prime}(t)$ are all positive for $t>t_{0}$.

No trajectory $C\left(v_{0}\right)$ can remain in II for arbitrarily large values of $t$.

No trajectory in I satisfies

(i) $x(t) \downarrow x_{0} \geqslant 0$ and $y(t) \uparrow \infty$ as $t \rightarrow \infty$, or

(ii) $y(t) \downarrow y_{0} \geqslant 0$ and $x(t) \uparrow \infty$ as $t \rightarrow \infty$, nor can any trajectory in I tend to a finite limit point $\left(x_{0}, y_{0}\right)$ in the closure of I as $t \rightarrow \infty$.

(D) No trajectory can go directly from II to I to II.

There remains the problem of translating these qualitative criteria into quantitative conditions on the coefficients of (2), the most difficult being (B). One set of conditions which can be shown to be sufficient is contained in the following

THEOREM. If the coefficients of (2) satisfy

(i) $c(t) \geqslant a(t)>0 ; b(t) \geqslant d(t)>0$;

(ii) $u^{\prime \prime}+\min \{b(t)-d(t), c(t)-a(t)\} u=0$ is oscillatory at $t=\infty$;

(iii) $\int^{\infty} t b(t) d t=\int^{\infty} t c(t) d t=\infty$;

then there exists a nontrivial solution $y(t), x(t)$ of (2) satisfying $y(\alpha)=$ $y^{\prime}(\alpha)=0=y(\beta)=y^{\prime}(\beta)$ for some $\beta>\alpha$.

When the conditions of this theorem are translated back to the original equation (1), they require that the coefficients $p_{2}(t)$ and $p_{0}(t)$ dominate 
$p_{1}(t)$ and $q_{1}(t)$ in an appropriate sense and that $1 / p_{2}(t)$ and $p_{0}(t)$ not go to zero too quickly. In the special case $p_{1}(t) \equiv 0, q_{1}(t) \equiv 0$, and $p_{0}(t)$ $<0$, our criteria are satisfied if

$$
\liminf _{t \rightarrow \infty}\left[t^{2} \min \left\{\frac{1}{p_{2}(t)},-p_{0}(t)\right\}\right]>\frac{1}{4},
$$

while Leighton and Nehari [6] essentially require

$$
\liminf _{t \rightarrow \infty} t^{2} \frac{1}{p_{2}(t)}>\frac{1}{4} \text { and } \liminf _{t \rightarrow \infty} t^{2}\left(-p_{0}(t)\right)>\frac{1}{4}
$$

A comparison of these conditions suggests that the criteria of the Theorem are reasonably sharp.

While the Theorem above establishes the existence of a conjugate point in the nonselfadjoint case, it does not provide explicit upper bounds for $\beta$. It is hoped, however, that these techniques may also prove useful in this regard.

\section{REFERENCES}

1. P. Hartman and A. Wintner, On disconjugate differential systems, Canad. J. Math. 8 (1956), 72-81. MR 17, 611.

2. W. T. Reid, Oscillation criteria for linear differential systems with complex coefficients, Pacific J. Math. 6 (1956), 733-751. MR 18, 898.

3. - A disconjugacy criterion for higher order linear vector differential equations, Pacific J. Math. 39 (1971), 795-806. MR 46 \#7632.

4. K. Kreith, Disconjugacy criteria for nonselfadjoint differential equations of even order, Canad. J. Math. 23 (1971), 644-652. MR 44 \#5557.

5. - A nonselfadjoint dynamical system, Proc. Edinburgh Math. Soc., 19 (1974), 77-87.

6. W. M. Whyburn, On selfadjoint ordinary differential equations of the fourth order, Amer. J. Math. 52 (1930), 171-196.

7. W. Leighton and Z. Nehari, On the oscillation of solutions of selfadjoint linear differential equations of fourth order, Trans. Amer. Math. Soc. 89 (1958), 325-377.

8. K. Kreith, A dynamical criterion for conjugate points, Pacific J. Math. (to appear).

DEPARTMENT OF MATHEMATICS, UNIVERSITY OF CALIFORNIA, DAVIS, CALIFORNIA 95616 